\title{
Management of a Heart Myxoma Through a Right Thoracotomy in a Patient With a Previous Sternotomy
}

\author{
Georgios P. Sotiropoulos ${ }^{\mathrm{a}, \text { e }}$, Maria P. Chounti ${ }^{\mathrm{b}}$, Panagiotis G. Hountis ${ }^{\mathrm{c}}$, \\ Konstantinos A. Plestis ${ }^{\mathrm{d}}$
}

\begin{abstract}
Myxoma is the most common benign cardiac tumor, presenting most frequently in the left atrium. Median sternotomy has been traditionally used to provide access to the heart and the mediastinal structures in cardiac operations. Recently, right thoracotomy and minithoracotomy have been used as an alternative. We present a case of a patient with left atrial myxoma, who had been subjected to coronary artery bypass graft 2 years ago. The previous full median sternotomy urged the need for another approach. A right lateral thoracotomy with one lung ventilation was chosen, offering excellent access to the heart and mediastinum. The patient was set under cardiopulmonary bypass and cardioplegia and the mass was excised through a left atrial incision. The perioperative course was free of complications. We suggest that right thoracotomy is a safe and efficient way of treating cardiac tumors in selected patients, when median sternotomy is considered complicated, not possible or contraindicated.
\end{abstract}

Keywords: Atrial tumors; Cardiac surgery; Right thoracotomy; Myxoma; Sternotomy

\section{Introduction}

Atrial myxoma is the most frequent primary tumor of the heart in adults. Left atrium (75\%) and right atrium (20\%) are the most usual presenting sites [1]. Median sternotomy is the main approach in the excision of atrial tumors. It offers great access to all the structures of the mediastinum and the lungs, enabling all types of cardiac operations as well as combined heart and lung operations [2]. Right thoracotomy is becoming a more popular approach, in selected cases, as surgeons are

Manuscript accepted for publication January 08, 2016

aNaval and Veterans Hospital of Athens, Athens, Greece

bNursing School, Technological Institute of Patras, Patra, Greece

${ }^{\mathrm{c}}$ Department of Cardiac Surgery, Iaso General Hospital, Athens, Greece

dLenox Hill Hospital, North Shore-LIJ Health System, New York, USA

e Corresponding Author: Georgios Sotiropoulos, Bouboulinas 47-49, Agia Paraskevi, Attica 15341, Greece. Email: sotiropoulos.georgios@outlook.com.gr

doi: http://dx.doi.org/10.14740/jmc2405w becoming more familiar with the technique. The reason for this trend is the possible benefits of right thoracotomy especially in redo sternotomy. A comparison of the two methods showed better postoperative results with the use of right infra-axillary minithoracotomy in terms of hospital stay, use of analgesics, postoperative bleeding and the need for blood transfusion [3].

\section{Case Report}

A 70-year-old male patient was referred to the cardiothoracic department due to the recent diagnosis of a left atrial myxoma. Main symptom was a progressively worsening dyspnea. An echocardiogram that was performed revealed a left atrial mass. Transesophageal echocardiogram confirmed the diagnosis. Tumor size approximated $3.5 \mathrm{~cm}$ (Fig. 1). His past surgical history included coronary artery disease and a coronary artery bypass graft $(\mathrm{CABG})$ through median sternotomy 2 years ago. Due to his previous surgical history, right thoracotomy was preferred to redo median sternotomy.

The patient was placed under general anesthesia with endotracheal double-lumen intubation, at left lateral position. A right lateral thoracotomy without rib excision was performed. The right lung was deflated and the pericardium was opened. Bypass circulation with fibrillation and direct cannulation of

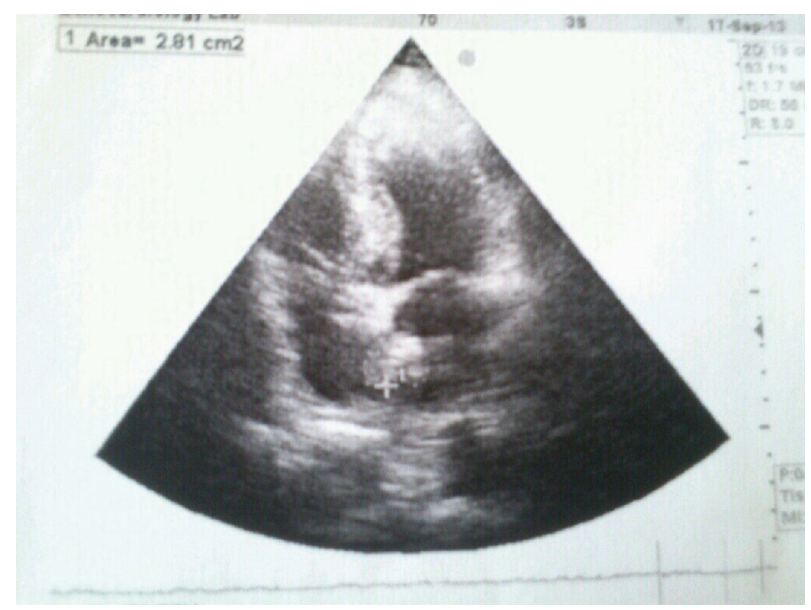

Figure 1. Heart ultrasound showing the left atrial mass attached to the interatrial septum. 


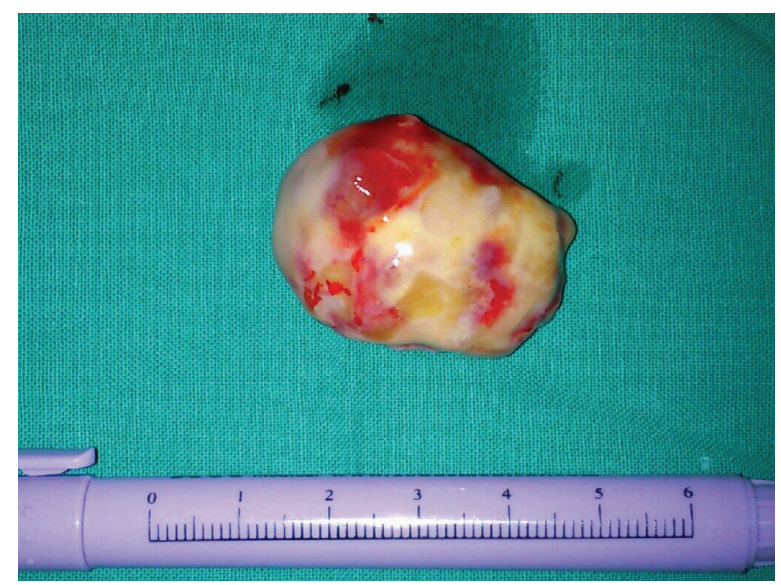

Figure 2. The mass completely excised.

the aorta and the inferior vena cava was initiated and blood cardioplegia was used. The left atrium was opened, revealing a pale mass (Fig. 2). The mass was attached to the interatrial septum. Complete excision of the mass was performed, including part of the interatrial septum (Fig. 3). A patch was used to secure the opening of the interatrial septum. Continuous prolene $3 / 0$ suture was used to close the left atrium and the bypass was terminated, after the transesophageal echocardiogram confirmed the lack of air in the cardiac cavities. The postoperative course was uncomplicated and the patient was discharged from the hospital after 5 days. Pathology examination confirmed the diagnosis of a myxoma.

\section{Discussion}

Full median sternotomy has proven its efficacy and safety as an approach for the excision of cardiac tumors [2]. Right thoracotomy has been reported as a safe method, with minimal complications for cardiac operations $[4,5]$ and most recently right mini-thoracotomy has achieved better results in terms of perioperative complications, hospital stay and need of pain control [2]. Furthermore, in selected patients, robotic cardiac surgery with right chest ports has been proven safe and effective in cardiac surgery [6]. The surgeon's experience and local guidelines are the main factors in choosing this approach. Furthermore, patient specific issues should be taken into consideration, such as past surgical history, as well as the patients wish for an acceptable cosmetic result.

In our case, previous full sternotomy for $\mathrm{CABG}$ that our patient had been subjected urged the need for another approach. The choice of right lateral thoracotomy through the fourth rib offered as excellent access to the mediastinum and the cardiac chambers, as direct cannulation of the aorta, superior and inferior vena cava, was feasible, with exceptional

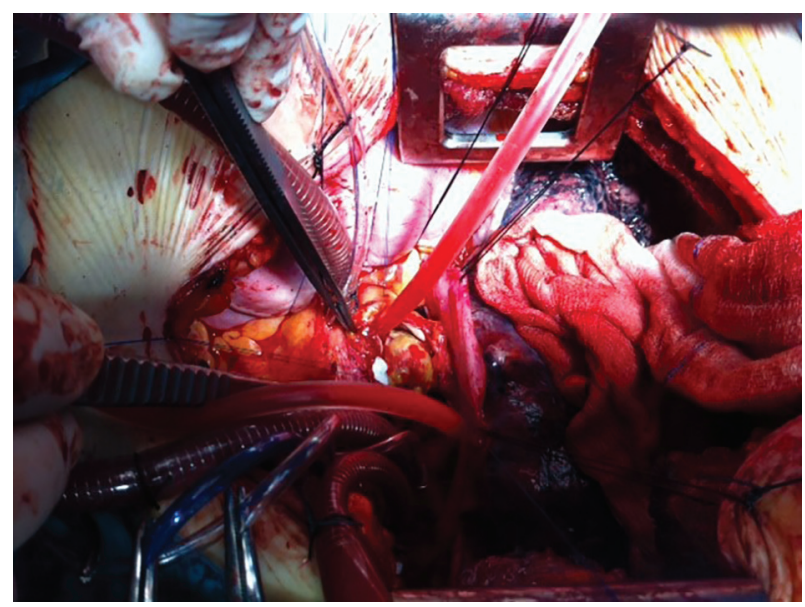

Figure 3. Intraoperative view showing the mass attached to the interatrial septum.

perioperative course. With this approach, we avoided possible complications of redo median sternotomy especially cardiac trauma, postoperative bleeding and distorted sternal closure or even sternal dehiscence.

We conclude that the use of right thoracotomy is a safe and effective way of treating patients who have been previously subjected to cardiac surgery and can be used as a standard procedure in the future.

\section{References}

1. Castillo JG, Silvay G. Characterization and management of cardiac tumors. Semin Cardiothorac Vasc Anesth. 2010;14(1):6-20.

2. Owais TA, Farber G, Garbade J, Mohr FW. Excision of a left atrial myxoma via a minimally-invasive technique: a possible routine access. Interact Cardiovasc Thorac Surg. 2011;12(5):875-877.

3. Tunerir B, Aslan R. An alternative, less invasive approach to median sternotomy for cardiac operations in adults: right infra-axillary minithoracotomy. J Int Med Res. 2005;33(1):77-83.

4. Grinda JM, Folliguet TA, Dervanian P, Mace L, Legault B, Neveux JY. Right anterolateral thoracotomy for repair of atrial septal defect. Ann Thorac Surg. 1996;62(1):175178.

5. Yang X, Wang D, Wu Q. Repair of atrial septal defect through a minimal right vertical infra-axillary thoracotomy in a beating heart. Ann Thorac Surg. 2001;71(6):20532054.

6. Gao C, Yang M, Wang G, Wang J, Xiao C, Wu Y, Li J. Excision of atrial myxoma using robotic technology. J Thorac Cardiovasc Surg. 2010;139(5):1282-1285. 